\title{
Navigating and Supporting Marginalized Identities in Dominant Pharmacy Spaces
}

\author{
Nicole D. Avant, PharmD, BCACP'; Rosalyn D. Davis, PhD, HSPP2 \\ ${ }^{1}$ University of Cincinnati Division of Experience-Based Learning and Career Education \\ ${ }^{2}$ Indiana University Kokomo Department of Psychology Clinical
}

\begin{abstract}
Addressing issues of diversity becomes more difficult when challenges impeding diversity efforts remain hidden. Issues like microaggressions, implicit bias, and lack of support are often present, but little is done by those in positions of power to mitigate or eliminate them. The purpose of this self-reflection is to address each of these areas and suggest six steps that may be taken to alleviate them: 1) anticipate microaggressions; 2) explore identities; 3) overcome internalized oppression and implicit bias; 4) recognize and mitigate stereotype threat; 5) differentiate yourself and provide space for others to differentiate themselves; 6) develop and reinforce support systems.

The initial phase of the manuscript focuses on implicit bias manifestations, specifically microaggressions, and how to prevent them from disrupting inclusivity. Following that will be a review of implicit bias, as well as the importance of self-awareness in promoting inclusivity in the workplace. Finally, the discussion will center what can be done do to create the best environment for ourselves and others as we move together towards creating environments that do more than speak to diversity efforts and are actually welcoming to each person within that space.
\end{abstract}

This manuscript is written for a few reasons. First, it provides insight on how to incorporate and integrate our multiple and sometimes divergent identities. Second, to discuss how our identities may influence how we react to diverse and non-diverse others. Third, this manuscript fills a gap in the field related to the experiences of those among us with multiple marginalized identities. This autoethnography seeks to empower not only myself but also others, especially those of us at the margins (e.g., people of color, women, the LGBT community) and those with dominant identities to speak up when they witness inequality.

Keywords: Microaggression, bias, privilege, minoritized, personal awareness, otherness, diversity and inclusion

I navigate this world as a black woman. I am acutely aware of the tendency of others to label black women as angry, aggressive, threatening, intimidating, and/or unprofessional. Consequently, I have muted and policed my emotions to avoid being stereotyped. I have also experienced guilt for feeling negative emotions. Over time, I have learned that I am human with a range of emotions, including anger, and I am allowed to feel without shame. It is absolutely justified to be angry when wronged. Now, I spend less time worrying about becoming a stereotypical persona and more time cultivating authenticity, freeing up time and energy to devote to tasks that are more meaningful.

I grew up in resource-deficient, segregated neighborhoods of Chicago making it extremely difficult to survive, thrive, and live healthy. As a child, I would flip through department store catalogues circling homes in the background where I wanted to one day reside. I wished I lived in a suburb just a few blocks away because it contained access to high-quality schools, wellfunded parks and recreation programs, and highly valued

Corresponding author: Nicole D. Avant, PharmD, BCACP Assistant Professor, University of Cincinnati

Division of Experience-Based Learning and Career Education 735K Joseph A. Steger Student Life Ctr, Cincinnati, Ohio 45221 Phone: 513-556-8254; Email: avantne@uc.edu homes. This suburb with access to robust community resources was predominately white. This variance in access is a classic example of structural racism, defined as normative, at times legalized, differential access to the goods, services, and opportunities of society by race. ${ }^{1}$ Although I had the good fortune to escape financially divested urban environments, as I climbed the mythical professional ladder, I found myself immersed in new dangers. That is, being the diverse other, only one, or one of few in dominant spaces exposed me to different forms of structural racism and other levels of racism.

To provide tips on establishing a successful career, I was invited by the University of Illinois at Chicago Student National Pharmaceutical Association to deliver a keynote at their annual banquet celebrating prospective pharmacy graduates. This invitation was a distinct honor as I graduated from this college with honors and many individuals from my support system would be in attendance. Therefore, in large part, it felt like returning home and providing a good service. I had committed to delivering an authentic talk, "I Didn't Hear a Word She Said Over the James Brown Wig": How to Be Heard When You are the Only One or One of Few, to students from diverse backgrounds on how to navigate their multiple, and often judged, identities Following that talk, a long-time mentor requested I write up the main, well-received, and much-needed points of my keynote to deliver to a broader audience. 
This manuscript is written for four primary purposes. The first is to provide guidance on how to navigate multiple marginalized identities. Navigating these identities is difficult but is often a much-needed skill set. My higher education and residency training have all occurred in historically white colleges and universities (HWCUs). Consequently, I have learned what the dominant culture celebrates and problematizes. Unfortunately, this knowledge forced me to shift my behavior accordingly. Shifting, or code switching, is a pressure to change oneself to survive, fit in, and make others comfortable. Many women of color shift (e.g., change their outer appearance, modify their speech) to navigate gendered racism especially during the hiring process. ${ }^{2}$ Shifting may also be known as "wearing a mask" or, as W.E.B. Dubois called it, double consciousness. ${ }^{3}$ While this method of coping helps people of color integrate into the systems that hired them, it is also emotionally and intellectually draining because most will never feel comfortable enough to stop switching.

Secondly, this paper seeks to discuss how to navigate our dominant identities. Although, I possess many marginalized identities as a fat, Black woman who grew up in an urban environment, I also possess many dominant identities. I am a college-educated, cisgender (my gender matches the sex assigned at birth), recently middle-class US citizen. Accordingly, I author this manuscript using experiences of my dominant identities to help ensure $I$ and others do not marginalize individuals further.

Thirdly, this manuscript highlights microaggressive experiences in pharmacy. The nature of microaggressions and implicit bias are still being fully understood when it comes to issues of diversity, training, hiring, and a multitude of other factors. Microaggressions can best be defined as repeated unintentional slights and indignities that people with marginalized identities experience. ${ }^{4}$ Those who deliver the microaggression often see it as a compliment and get defensive when it is addressed, making it difficult to stop them from occurring. Implicit or unconscious bias, though well studied is also a concept not willingly accepted by individuals who often see themselves as embracing culture and diversity ..$^{5-6}$

Lastly, this manuscript fills a gap in published experiences of those with marginalized identities. This autoethnography seeks to empower not only myself but also others, especially those of us at the margins (e.g., people of color, women, the LGBT community) and those with dominant identities to speak up when they witness inequality. This reflection cannot address every experience of multiple diverse identities, but hopes to be transparent in conveying the experience of a black woman in a field still learning to successfully navigate diversity.

Six recommendations were provided to the audience to navigate their identities. One, anticipate microaggressions. Two, explore your identities. Three, overcome internalized oppression and implicit bias. Four, recognize and mitigate stereotype threat. Five, differentiate yourself and provide space for others to differentiate themselves. Six, develop and reinforce support systems.

My first piece of advice for students was to anticipate microaggressions. This recommendation may defuse some of the shock and awe when they inevitably arise, along with being prepared with a tactful response, when possible. This is one of those moments when being able to fully employ double consciousness may be crucial to maintaining status within places of employment.

In 2015, I eagerly accepted a faculty appointment focused in diversity and inclusion. I learned later that I would be the first Black woman and the only Black faculty member at the College. While so many of my friends and family were excited at my trailblazing, I felt anxiety. I knew all too well the simultaneous feelings of hyperinvisibility and hypervisibility at a HWCU. People taking notice when you miss a meeting, but ignoring your contribution to the meeting. Or, colleagues who are slow to recognize your achievements, but quick to point out errors. I had already experienced the weight of what I now know as microaggressions. The importance of preparing for these is likely overlooked in our efforts to prepare for everything else being the first and only of an identity group.

Microaggressions can be interpersonal or environmental. ${ }^{4} \mathrm{An}$ interpersonal microaggression includes repeatedly asking Asian individuals where they are from and not accepting their first typically US-based response. The underlying implication is that these individuals are not "really American" because they do not possess blue eyes or blond hair. If the Asian colleague responds with frustration or exasperation, they may be told they are being sensitive or overreacting. Even though they are justified in their reactions, they may start to second guess themselves and begin to stifle their reactions in the future, which impacts their work relationships, as well as their mental health. ${ }^{7}$

Alternatively, in a search committee meeting there may be repeated refrains that "the most qualified person should get the job," when the discussion is specifically targeting racially diversifying leadership. The implicit message is that people of color will likely not be the most qualified for the job and their token presence is not desired. This overlaps with issues of bias that requires more focused inclusive leadership development for those in hiring positions, as well as those that oversee new hires structurally. ${ }^{8}$

An example of an environmental microaggression could be the exclusion of underrepresented groups in public spaces. That can include books, statues, employees, movies, advertising, accepted standards of beauty and talent, and naming of buildings. When those situations are rectified, the reaction of the dominant group in attacking the item is also another attack on the group and attempts to both demean and diminish the enjoyment of the product (i.e., "Why is there a show called The 
Wiz? White people could never get away with that" - all while ignoring the source material in The Wizard of Oz).

In general, the intent of the microaggression is to be complimentary. The fact that the aggressor hasn't considered the impact of the statement is the heart of why it needs to be prepared for as much as possible. If it sounds like one cannot take a compliment, that adds to the layer of misinformation already likely floating around about the target. In a marginalized status, there is not room for the misinformation to stack up because it will eventually be deemed a performance or personality issue that may assist in the exit of diverse employees.

Usually victims of microaggressions either avoid them or fight every last battle. ${ }^{9}$ Fighting every battle may exhaust and alienate individuals from others. Still, ignoring the impact of microaggressions is not good for one's health. One must learn to let out frustrations incrementally through various means, such as exercising, talking with friends, and seeking therapy instead of allowing them to buildup. If one's response is "out of control", those around may not categorize the reaction as a direct reflection of the repeated assaults one has experienced. They may characterize the reaction as an overreaction to such a "small" slight, ignoring the cumulative impact of these "small" slights.

Considering my multiple, intersecting marginalized identities, I must become a conflict resolution superhero. For example, it is common for my contributions to be ignored after speaking up at a meeting, but then someone else is subsequently rewarded for expressing the same idea in the same meeting. I have decisions to make each time a microaggression occurs. I have to decide if it is worth pulling or pushing back. ${ }^{9}$ I ask myself who holds the power and complete a cost-benefit analysis. Does the cost of expressing my feelings outweigh the benefits? If I decide the cost is too great or to pull back, I have learned the importance of releasing that energy in other places to minimize the impact on my emotional and physical health. If I decide the benefit exceeds the cost or to pushback, then I must respond strategically to interrupt microaggressions.

I attended a national conference during a recent summer and a fellow academic in a session said the reason Africans are being hired over African-Americans in Colleges of Pharmacy is because "they work hard." The coded message was that African-Americans are lazy. As an African-American woman, this was not the first time I had heard the stereotype and yet I had not prepared for such a hurtful false narrative perpetrating the myth of meritocracy at a national meeting.

It is important to prepare for microaggressions before they arise, not only as the victim, but also as a third party. Years ago, I participated in a college meeting and a senior white faculty member turned and asked me publicly, the only black faculty member at the College, "Why is diversity even important?" । didn't owe him a significant or substantial response, but I responded. The other attendees in the meeting had normalized his response by staying silent; no one challenged the question. I received a few emails afterwards expressing their dismay and applauding my response. Although they were attempting support, I was hurt they didn't speak up in the moment. I already felt disempowered as junior faculty and had anxiety due to the heightened visibility of being the only black face in the College.

When no one spoke up, I thought Does everyone share the same thoughts? If they disagree, why didn't they ask questions to challenge the behavior and show me support? Someone could have simply said, "ouch" to let me and others know that I wasn't the only one impacted by this question. Instead, the room was silent and the onus to educate those with dominant identities was on me, someone from the targeted group. Microaggressions are exhausting and harmful enough. Hence, support and reinforcement are needed from others, especially in the moment. The support is needed to avoid alienating those who may already feel like they are on the outside trying to navigate a system that was not designed with them in mind.

I fully recognize that interrupting microaggressions is easier said than done; I have not always followed my advice. I was in a meeting with a colleague who was speaking negatively of a presenter who possessed an accent. She did this in the presence of another colleague who possesses the same accent. Instead of saying something as simple as "ouch", I sat there in silence while my colleague was being ostracized and marginalized. I knew what it felt like to be targeted in a meeting with no one intervening and yet I did not intervene. We must use our dominant identities to support others with marginalized identities even when it is uncomfortable. The goodwill engendered in those moments far outweighs the negativity that will ensue by doing nothing. Silence may be seen as agreement or complicity.

The second tip given was to explore your identities. Knowing areas of growth or addressing potential blind spots allows individuals to contribute to more open discussions about issues of diversity and inclusion. ${ }^{10}$ In order to promote inclusivity in our organizations, it is important to develop self-awareness both as employees and as an organization. We cannot live up to ideals that haven't been clearly stated or if we continue to allow people to not demonstrate commitment to those ideals. I have started developing personal awareness related to my identities to minimize marginalizing others. I have two recommendations for those who wish to do so.

First, identify the dominant/privileged and marginalized groups in our society and engage in social identity mapping to determine where you hold dominant and marginalized identities. Many identities have been deemed by our society as superior or inferior. For example, privileged groups in our society include men, white persons, heterosexual people, the 
middle-upper class, Christians, US born citizens, able-bodied persons, thin individuals, European ethnicities, and those with strong English literacy. ${ }^{11}$ That list is not exhaustive and depending on your perspective may not be completely valid, but those groups tend to experience less explicit discrimination for simply existing. Revisit the list you ultimately create from time to time and see if things have changed. For example, as you age, you may lose the perception of intelligence because you are young, but may gain the belief that you are now wise. These identities are not existing in a bubble, but working collaboratively to shape your experiences.

Second, pay attention to common characteristics of dominant and marginalized identities. For example, when individuals inhabit marginalized identity positions, they may be subjected to a tsunami of burdensome emotions, all while seeing the dominant group as unaware of their daily realities. ${ }^{4}$ They may feel angry, hopeless, and disempowered. They may experience the "cumulative impact" of hiding their emotional reactions to microaggressions. They may also internalize negative stereotypes about their identity group. ${ }^{1}$ All or none of those things can happen as they move through spaces where their other status is always present. They must protect themselves when possible, seek support from others in similar positions, and provide support when asked.

Although it may be easy to reflect on one's marginalized identities, it is important to also reflect on the characteristics associated with the dominant/power/privileged identities they may inhabit to ensure they are not marginalizing others. For example, white people tend to feel attacked, silenced, guilty, insulted, scared, and/or angry when their assumptions regarding race are challenged. ${ }^{12}$ They may then emotionally withdraw or focus on their intentions versus the impact of their actions. They may make dismissive claims to justify their actions such as, "I know people of color", "I can't say anything right!", or "That was not my intention". These assumptions, attitudes, and behaviors may be applied to other dominant identities.

Returning to the encounter with my colleague with the accent, I later apologized to her for not speaking up in the moment. She said she decided not to continue the relationship with the perpetrator of the microaggression. I almost dismissed her feelings by telling her, "But she (perpetrator) is a really good person." However, I quickly recognized how I was exhibiting a dominant group characteristic. When I shared with my senior colleague that I was shocked by that question of "Why is diversity even important", I was instructed to ignore it because this person was a good person. My feelings were completely dismissed and yet I almost dismissed the feelings of my colleague. It is imperative to recognize our identities and common characteristics associated with our identities to ensure we are not marginalizing others. I encourage us, especially when we are operating from our dominant/power identities, to take the time to listen to folks from marginalized groups. They are the expert in their experiences and identities.
Moving to the third recommendation overcome implicit biases and internalized oppression. Overcoming these items is a process and a goal. I am working towards overcoming internalized inferiority in my marginalized identities and overcoming internalized superiority in my dominant identities. While crucial for inclusivity, it will take consistent and persistent intentional actions to undo. It involves many actions including critically evaluating bias in media messages. Are women usually engaging in household activities instead of serving in leadership? Are older adults usually shown as infirm and forgetful instead of competent individuals? Are black persons usually casted as criminals or athletes instead of engaging in high academic achievement? Are Muslims usually shown as terrorists instead of protagonists?

Most of the professors I see on television and who have instructed me are white men. As a result, my brain has likely made an implicit association, professors equate to white men. This implicit association contributes to feelings of imposter syndrome in the academy, as my identities do not align with my implicit association. Consequently, when my behaviors and language do not align with the way I think professors should act and speak, it adds to my insecurities. I have to continually pushback on these associations through interacting with more black women professors. I continually define and redefine how black women professors should act and speak. I am actively working to deprogram my brain of years of stored implicit associations. This is something that will not occur overnight but is definitely worth the effort it takes to focus less on feelings of inferiority and more on affirming my "black girl magical" self.

I recall befriending a black woman a few years ago. When she told me she was an engineer, I remember being shocked. I felt uncomfortable in my shock and immediately recognized my implied association for "white men equates to engineer." If we do not take the time to identify and overcome our implicit preferences, they may manifest as microaggressions.

Implicit biases can be identified through Harvard Implicit Association Tests (IAT). ${ }^{6,13}$ Consequently, once implicit biases have been identified, it is important to take action to counter them.

Overcoming internalized oppression involves more work than just stating that it should be done. Individuals must address the numbers and variety of ways in which you have been told you are less than and incapable or unwanted by the dominant group. Think of young black children who are brimming with enthusiasm and show sparks of giftedness. Now think of the number of times their dreams are crushed either because the school is underfunded or their teachers don't believe they are bright enough. ${ }^{14}$ By the time that student reaches their senior year, the culmination of messaging they are not as good as their counterparts becomes a message they haven't even begun to unpack. Furthermore, it can taint all future accolades as "flukes" or received for a reason other than merit. This is more than figuring out one is not an imposter who has claimed a 
space that was not designed for them, but requires people of color, in particular, to learn about themselves, their culture, and their own strengths in a way that flies in the face of their alleged deficits.

We also have to recognize and mitigate stereotype threat. Stereotype threat is fear that an individual will confirm a stereotype. ${ }^{15}$ That threat may make the stereotyped group member hypervigilant regarding their behaviors to avoid being stereotyped. However, diligent behavioral checking may also make them uncomfortable, less genuinely themselves, and more susceptible to stress while they perform a delicate dance of avoidance.

Although I am aware of this phenomenon and am working to pushback on bias, I still experience stereotype threat. I waste energy on avoiding confirming stereotypes instead of using the energy to improve my performance. Ironically, in trying to avoid confirming a stereotype, I, like others, am more likely to confirm them due to increased anxiety, stumbling over my words, and so forth. I remember experiencing this vividly during clinical rotations and sometimes it still shows up during personal and professional interactions.

Fortunately for me, even though I did not possess the language to describe what was happening to me until recently, I had strong mentors in pharmacy school and in my pharmacy residency program that mitigated, likely without knowing it, stereotype threat. They invoked high standards and assurance that I could meet those standards. ${ }^{15}$ This was crucial as I came into pharmacy school with insecurities related to my marginalized identities. I attended undergraduate and pharmacy school as well as residency training at HWCUs, which were identity-threatening environments (e.g., low students and faculty of color representation ). But, I had others who affirmed me. I didn't think I wrote well and I expressed my fears to one of my white mentors. He told me that my writing was fine and I felt more confident in my writing thereafter. I felt I didn't interview well, but my white residency program director told me I was one of the strongest interviewers he encountered and my interviewing confidence peaked. Without those mentors, I may have abandoned my aspirations of working in the academy.

I cringe in writing that white people should affirm people of color. However, for some, that validation may be the only affirmation that is accepted until they begin to have more confidence in themselves. Additionally, it can relieve the extra emotional labor of faculty of color, if white faculty members can also fulfill supportive roles. Over time, I have learned to not pathologize my identities. However, it is a work in progress and individuals may not be there yet. Thus, it is important to uplift others who are both alike and dissimilar to us.

In addition to setting high standards and assurances to meet those standards, other ways to minimize stereotype threat include creating identity safe environments, education regarding stereotype threat so individuals can name their experiences, encouraging individuals to externalize negative thoughts, and guiding individuals to engage in self-affirmation exercises. ${ }^{15}$ Self-affirmations are ideal, but sometimes affirmations from those with dominant identities is helpful, especially when those with marginalized identities have internalized their inferiority.

That support is critical and it must allow space to differentiate yourself and provide space for others to differentiate themselves. Especially considering some identity groups are underrepresented in pharmacy, appreciating our differences is vital to inclusivity and retention. I am learning to view my most salient, marginalized identities as strengths. My interlocking experiences related to my identities afford me unique perspectives. For instance, I grew up in resource-deficient neighborhoods but now I reside in resource-dense neighborhoods. I can easily and intimately compare and contrast community resources such as schools, housing, food choices, public safety, parks and recreation, businesses, and political clout. ${ }^{16}$ The way I describe these social determinants of health and social determinants of equity using personal stories in the classroom and during speaking engagements resonates well with attendees.

My life experiences are distinctive strengths that contribute to meeting organizational goals. I served as a collaborator on a $\$ 2.5$ million grant sponsored by the Office of Minority Health's Department of Health and Human Services to build pipelines from urban schools to the University of Cincinnati where I inimitably connected with students. It is important to appreciate, not problematize, our different identities and experiences.

I created a personal mission statement during my undergraduate years to increase the upward mobility of others through academics, economics, and health. In centering underrepresented groups, I have created a niche in health at the intersection of what I love (passion), the profession needs (service gap), the profession will reimburse (career), and I do well (skill sets). I effectively teach heath disparities, inclusive leadership, and social determinants to students and clients to cultivate structurally-competent clinicians. My research focuses inclusion (i.e., implicit bias in pharmacy, microaggressions in pharmacy, pushing for health equity through implicit bias and structural competency pharmacy education). ${ }^{6}$ My expertise continues to grow in a direction that supports my mission statement, passion, entrepreneurship, career, and skills.

Uplifting, centering, and amplifying underrepresented groups have not always been easy as employers and colleagues have, at times, devalued its importance. However, I continued to identify a gap in the health professions related to my passion 
and unique talents. Now, a demand exists from medical centers and academic institutions for my expertise.

Cultivating and appreciating differences at the individual level is important but we should also be open to systemic changes. ${ }^{17}$ We should ask ourselves: What groups hold the power at our institutions? How long have these groups held the power? Are we centering the voices of marginalized groups? Are we listening to their perspective? Are we forcing assimilation or acculturation? Are we contributing to the marginalization of others? Are we unintentionally or intentionally excluding others? Are we allowing space for structural changes? What groups of people are we devaluing, ignoring, and excluding? Why? What are their intersectional identities? We need to be intentional about aligning our organizational mission with the talents of our diverse communities. And if we are missing or invalidating perspectives, we need to ensure we capture those perspectives.

Right before I graduated with my undergraduate degree, I was debating if I should stop treating my hair with harmful chemicals to straighten it. If I stopped introducing harmful chemicals to my body, would I be able to receive gainful employment from those who may deem the way my hair naturally grows out of my scalp as unprofessional? If I continued to introduce harmful chemicals, then I may be able to infiltrate the system to make changes. I decided to stop relaxing my hair. Two decades later, I am still engaging in balancing acts of authenticity and challenging the status quo with maintaining my career. Pushing for institutional change isn't easy but necessary for the continued growth of our work places, our profession and our colleagues-both present and future. Hence, making spaces for unique identities and perspectives must be valued.

My final suggestion is to develop and reinforce support systems. Most troubling is that even when the educational attainment is the same, some marginalized groups still experience health disparities due to the stress related to discrimination. Individuals must recognize that they cannot navigate these identities alone especially when their mere presence and work as status quo disrupters are seen as divisive. Pushing for inclusion can be lonely and stressful enough. Thus, it is absolutely necessary to develop systems to overcome psychosocial stressors related to interpersonal, internalized, institutionalized inequities, as well as historical traumas and anticipatory stress. ${ }^{18}$

Many ways exist to create a much-needed support system. First, find mentors. Everyone should have more than one mentor to support them in different areas. Those with marginalized identities may have an added burden to find mentors who can relate to the intersection of their identities. However, whomever they choose to, and that agrees to fulfill that role for them, should be someone that actively believes in their talents, will not doubt their experience, and is able to navigate them through the challenges they have to face.

Second, they should network and surround themselves with powerful folks. Being a status quo challenger can leave one vulnerable. They need the support of others. Develop a squad or a village to maintain health, be uplifted, encourage taking breaks, and recognize when "you are doing the most". Participate in or create, if needed, an affinity group, preferably funded by the company or organization. This group does not have to be official, but it does need to be an active source of support.

Third, build coalitions with others, particularly other marginalized groups. It is important that there is not a fight over resources but instead a fight for more resources. Fourth, mentor others. "Lift while you climb". I am only here because of others. I stand on the shoulders of my ancestors.

When we operate from our dominant identities, it is important to allow others to have these groups without feeling excluded. Marginalized groups would not need these spaces, if default groups did not exist in spaces. Thus, it is important for us to allow these spaces.

In summary, my learning of white culture usually has been unidirectional. I have learned the nuisances of their culture, but usually they do not take the time to learn my cultural nuisances. Thus, I shift. I have shifted my outward presentation from the way I styled my hair to the way I inflected my voice to ensure I would not be ostracized. I assimilated to move my career forward. While I found shifting necessary for professional success, it was not without inherent risks.

As I have climbed the mythical "ladder for success," I have learned to shift less to improve my health. However, I have found that although I feel more liberated in being my authentic self, it makes others more uncomfortable. Making those with dominant identities uncomfortable may negatively impact my career trajectory, creating a "catch 22." Do I choose authenticity, prioritizing my heath, but at the expense of others' comfort? Or, do I continue to shift to make others who hold power comfortable, prioritizing my career, but at the expense of my health? These dilemmas could be minimized, if dominant groups cultivated spaces for diverse others to be their authentic selves without harm.

Many of our organizations have language related to diversity and inclusion in our mission statements. To move from ideology to reality, we need to be intentional about creating a space to support marginalized groups. It calls for all of us to explore our identities, anticipate microaggressions, overcome internalized oppression and implicit bias, as well as develop support systems. We need to listen to diverse others when they speak to the culture of our organizations and we need to be committed to doing the hard work of advancing forward as a 
unit. Every one of the suggestions may not resonate with every person that reads this manuscript. My hope, however, is that it gives students, faculty, and organizations a place to begin discussions about what it is they need to become the ideal versions of themselves.

Acknowledgements: The primary author acknowledges Dr. Popovich, University of Illinois at Chicago College of Pharmacy; Dr. A. Littisha Bates, University of Cincinnati College of Arts and Sciences; Dr. Leila Rodriguez, University of Cincinnati College of Arts and Sciences; and Dr. Megan Lamkin, University of Cincinnati Experience-Based Learning and Career Education for their advising and editing. The primary author would also like to acknowledge the Social Justice Training Institute and Mr. Brice Mickey for holding spaces that allow introspection.

Conflicts of Interest: The primary author engages in consulting work and diversity training for anchor institutions such as academic health and medical centers through Avant Consulting Group. I declare no other conflicts of interest or financial interests that the authors or members of our immediate families have in any product or service discussed in the manuscript, including grants (pending or received), employment, gifts, stock holdings or options, honoraria, consultancies, expert testimony, patents and royalties.

\section{References}

1. Jones CP. Levels of racism: A theoretic framework and a gardener's tale. American J Pub Health. 2000; 90 (8):1212-1215. doi: 10.2105/AJPH.90.8.1212.

2. Jones $C$, Shorter-Gooden K. Shifting: The Double Lives of Black Women in America. 1st ed. New York: HarperCollins; 2003.

3. Du Bois WEB. The Souls of Black Folk. New York: Dover Publications; 1903.

4. Sue DW, et al. Racial microaggressions in everyday life: Implications for clinical practice. Am Psychologist. 2007;62(4):271-286 doi: 10.1037/0003-066X.62.4.271

5. Sukhera, J, Watling, C. A framework for integrating implicit bias recognition into health professions education. Acad Med, 2018 93(1):35-40. doi: 10.1097/ACM.0000000000001819

6. Avant ND, Weed E, Connelly C, Hincapie A, Penm, J. Qualitative analysis of student pharmacists' reflections of Harvard's Race Implicit Association Test. Curr Pharm Teach Learn, 2018; 10(5): 611-617. doi: 10.1016/j.cptl.2018.02.002.

7. Williams DR, Williams-Morris, R. Racism and mental health: The African American experience. Ethn Health, 2000; 5(3/4), 243-268. doi: 10.1080/713667453.
8. Segrest Purkiss SL, Perrwe' PL, Gillespie TL, Mayes BT, Ferris G. Implicit sources of bias in employment interview judgements and decisions. Org Beh Human Dec Proc, 2006; 101(2): 152-167. doi:10.1016/j.obhdp.2006.06.005

9. Rockquemore, KA. Pick Your Battles. Faculty Diversity. https://www.facultydiversity.org/mondaymotivator/pickyourbattles. Published April 2018. Accessed November 122018.

10. Ratts MJ. Charting the center and the margins: Addressing identity, marginalization, and privilege in counseling. J Ment Health Couns, 2017; 39(2): 87103. doi: 10.17744/mehc.39.2.01.

11. Adams M. Readings for Diversity and Social Justice. Third ed. New York: Routledge Taylor \& Francis Group; 2013.

12. DiAngelo RJ. White Fragility: Why it's so Hard for White People to Talk about Racism. Boston: Beacon Press; 2018.

13. Nosek BA, Smyth FL, Hansen JJ, et al. Pervasiveness and correlates of implicit attitudes and stereotypes. Eur Rev Soc Psychol. 2007; 18: 36-88. doi: 10.1080/10463280701489053.

14. Grissom JA, and Redding C. Discrimination and disproportionality: Explaining the underrepresentation of high-achieving students of color in gifted program. AERA Open, 2016; 2(1): 1-25. doi: $10.1177 / 2332858415622175$.

15. Burgess DJ, Warren J, Phelan S, Dovidio J, van Ryn M. Stereotype threat and health disparities: what medical educators and future physicians need to know. J Gen Int Med. 2010; 25(S2): 169-177. doi: 10.1007/s11606-009-1221-4.

16. Jones CP, Jones CY, Perry GS, Barclay G, Jones CA. Addressing the social determinants of children's health: a cliff analogy. J Health Care Poor Underserved, 2009; 20(S4):1-12. doi: 10.1353/hpu.0.0228.

17. Tomlinson Y, Baruch M. Framing Questions on Intersectionality. US Human Rights Network. 2013. https://www.ushrnetwork.org/sites/ushrnetwork.org /files/framing questions on intersectionality 1.pdfA ccessed October 2, 2018.

18. Williams DR, Mohammed SA. Discrimination and racial disparities in health: evidence and needed research. J Beh Med. 2009; 32(1):20-47. doi: 10.1007/s10865-008-9185-0. 